\title{
Change of Parameters of the Koiwa-Hasiguti Dynamic Dislocation Relaxation in Nanostructured and Polycrystalline Zirconium after Severe Plastic Deformation and Annealing
}

\author{
P. P. Pal-Val $\left({ }^{1}{ }^{1}\right.$ L. N. Pal-Val, ${ }^{1}$ A. P. Rybalko, ${ }^{2}$ and E. N. Vatazhuk ${ }^{1}$ \\ ${ }^{1}$ B. Verkin Institute for Low Temperature Physics and Engineering, National Academy of Sciences of Ukraine, Nauki Ave. 47, \\ Kharkov 61103, Ukraine \\ ${ }^{2}$ S. Kuznets Kharkov National University of Economics, Nauki Ave. 9a, Kharkov 61166, Ukraine
}

Correspondence should be addressed to P. P. Pal-Val; palval@ilt.kharkov.ua

Received 9 October 2017; Accepted 13 December 2017; Published 19 March 2018

Academic Editor: Philip Eisenlohr

Copyright (c) 2018 P. P. Pal-Val et al. This is an open access article distributed under the Creative Commons Attribution License, which permits unrestricted use, distribution, and reproduction in any medium, provided the original work is properly cited.

The temperature dependences of acoustic properties of nanostructured and polycrystalline zirconium are investigated in the temperature range of $100-340 \mathrm{~K}$. The effect of severe plastic deformation and subsequent annealing on key parameters of the Koiwa-Hasiguti acoustic relaxation in zirconium is studied in detail. It is established that, due to intensive plastic deformation, the relaxation strength considerably increases, and the temperature and the width of the corresponding relaxation peak systematically decrease with reduction of the mean grain size in the samples. Annealing leads to a partial recovery of the relaxation strength and the peak temperature back to the initial values in undeformed samples, but the width of the relaxation peak shows an additional decrease. The majority of the effects observed can be explained by changes in dislocation subsystems of the samples during intensive plastic deformation and annealing. An influence of a random scatter of the relaxation time on the main parameters of the Koiwa-Hasiguti peak is established using the statistical analysis based on the lognormal distribution. It is shown that the parameter $\beta$ of the lognormal distribution determines the width, height, and asymmetry of the peak and also allows estimating the relaxation strength from the peak height. An algorithm for retrieving the parameter $\beta$ from experimental data is presented.

\section{Introduction}

The study of physicomechanical properties of high-fragmented polycrystals with the mean grain size of order $100 \mathrm{~nm}$ is of interest both from the fundamental and technological points of view. Fragmentation of polycrystalline metallic materials and their transition into the nanostructured (NS) state change essentially such important physical and technological characteristics as elastic moduli, strength and plasticity, the Debye temperature, the Curie temperature, corrosion resistivity, etc. In some cases, NS metals have much higher operational characteristics in comparison with coarse-grained polycrystalline metals and may be regarded as perspective constructional materials.

Severe plastic deformation (SPD) is one of the simplest and accessible methods of obtaining high-fragmented metals. High levels of plastic deformations may be achieved using equal-channel angular pressing, high-pressure torsion, accumulative roll-bonding, hot and cold rolling, drawing, hydroextrusion, forging, and the variety of their combinations $[1,2]$. SPD methods allow obtaining large and practically pore-free bulk samples that are not accessible when using other techniques, for example, by the compaction of superfine powders. However, samples prepared with the help of the SPD techniques are not in thermodynamic equilibrium due to a huge number of deformation defects, first of all, high density of dislocations. This can be considered as one of the main reasons for the significant change of physicomechanical properties of polycrystals during formation of the NS states as well as at subsequent thermal and/or mechanical treatments. Instability of the NS materials is a serious restriction for their wide application as elements of constructions which are subjected to extreme working loads and temperatures. Therefore the study of changes in microstructures of the NS metals and alloys at 
TABLe 1: Preparation schedules and basic characteristics of the samples.

\begin{tabular}{|c|c|c|c|c|}
\hline Sample & $\bar{d}, \mu \mathrm{m}$ & $k_{v}$ & Preparation scheme & Post-SPD annealing \\
\hline $\mathrm{Zr}-\mathrm{CG}[7]$ & 150 & 0.3 & A $(1073 \mathrm{~K}, 4$ h. $)$ & - \\
\hline Zr-HEA & 12 & 0.85 & $\mathrm{HE}(4.1)+\mathrm{A}(853 \mathrm{~K}, 1 \mathrm{~h})$. & - \\
\hline Zr-HEAD & 2 & 0.71 & $\mathrm{HE}(4.1)+\mathrm{A}(853 \mathrm{~K}, 3 \mathrm{~h})+.\mathrm{D}(0.56)$ & - \\
\hline $\mathrm{Zr}-0$ & 0.392 & 0.66 & $\mathrm{HE}(4.1)+\mathrm{A}(853 \mathrm{~K}, 3 \mathrm{~h})+.\mathrm{D}(2.4)$ & A $(425 \mathrm{~K}, 1$ h. $)$ \\
\hline Zr-01 & 0.117 & 0.35 & $\mathrm{HE}(4.1)+\mathrm{D}(2.4)$ & A $(425 \mathrm{~K}, 1$ h. $)$ \\
\hline $\mathrm{Zr}-02 \mathrm{RT}$ & 0.113 & 0.32 & $\mathrm{HE}(1.1)+\mathrm{CD}(4)+\mathrm{D}(2.4)$ & A $(425 \mathrm{~K}, 1$ h. $)$ \\
\hline Zr-02CE & 0.100 & 0.28 & $\mathrm{HE}(1.1)+\mathrm{CD}(4)+\mathrm{D}(1.4)+\mathrm{CE}(1.0)$ & A $(425 \mathrm{~K}, 1$ h. $)$ \\
\hline
\end{tabular}

A means annealing (annealing temperatures and its duration are given in parentheses), HE means "hot" extrusion at the temperature $795 \mathrm{~K}$, D means drawing at room temperature, $\mathrm{CD}$ means cyclic deformation at room temperature, $\mathrm{CE}$ means cryoextrusion at $77 \mathrm{~K}$, and $k_{v}$ is the coefficient of variation, characterizing homogeneity of a grain-size distribution. In parentheses, the values of the "true" plastic deformation $e$ are presented (Section 2 in the text).

various stages of their preparation and post-SPD treatments is an important and actual problem in modern materials science and technology.

Experimental study of elastic and inelastic properties of the NS metals in a wide temperature range using acoustic spectroscopy methods may provide important information on dynamic properties of dislocations in metals. These methods offer nondestructive way of obtaining elastic and inelastic characteristics of materials, possess high structure sensitivity, selectivity and reproducibility. In the present work, acoustic properties of intensively deformed zirconium are investigated in temperature range $100-340 \mathrm{~K}$. Thanks to a number of important physical parameters and their optimal for practical applications combination (resistance to radiating damages, small cross section for scattering of thermal neutrons, plasticity and strength, high corrosion stability) zirconium finds wide application in nuclear power engineering. It was established that the SPD fragmentation of $\mathrm{Zr}$ polycrystals essentially improves physicomechanical characteristics of pure zirconium, especially, in the range of low temperatures [3]; however, the evolution of parameters of dislocation structure at different stages of the preparation and post-SPD processing of NS zirconium was not investigated in detail.

The main purpose of the present work is a comparative study of dislocation structure evolution in the pure zirconium subjected to various SPD manufacturing schemes and post-SPD annealing by investigation of changes in the main parameters of the low-temperature Koiwa-Hasiguti $(\mathrm{KH})$ dislocation acoustic relaxation.

\section{Experimental}

Samples of polycrystalline iodide zirconium were studied. The starting material was subjected to double electron-beam remelting. The grain size in the initial ingots was $\sim 1 \mathrm{~mm}$, and the integral purity of the material was characterized by the relative change of the resistivity between $293 \mathrm{~K}$ and $4.2 \mathrm{~K}$ $\rho_{293} / \rho_{4.2} \approx 40$. Fragmentation of the grain structure of the samples was achieved during SPD on several technological schemes. The value of plastic deformation at extrusion, drawing, upsetting, and squeezing was characterized by the value of the "true" plastic deformation $e=\ln \left(S_{0} / S\right)$, where $S_{0}$ and $S$ are the initial and final cross sections of the samples.
The use of the multistage SPD schemes was aimed both at reducing the mean grain size $\bar{d}$ and achieving a more uniform grain-size distribution and a higher degree of the grain equiaxiality. The homogeneity of the grain-size distribution was characterized by the coefficient of variation $k_{v}=s / \bar{d}$, where $s$ is the standard deviation from the value of $\bar{d}$. The mean grain size and other characteristics of the structure of samples were determined by means of histograms. Details of the sample preparation procedure and methods for determining their structural characteristics are described in [4-6], and the main characteristics of the samples studied are given in Table 1.

Acoustic measurements were carried out by the twocomponent composite vibrator technique with piezoelectric excitation [8]. Longitudinal standing waves were excited in the samples at the fundamental frequency $f \sim 73 \mathrm{kHz}$ and also at the 3rd and 5th harmonics of the quartz transducer ( $\sim 220$ and $\sim 365 \mathrm{kHz}$, resp.). The samples were cut from the initial billets using spark erosion cutting. Then the end faces of the samples were polished with abrasive materials to achieve the required length $(\sim 30 \mathrm{~mm})$, flatness, and parallelism.

The measurements were carried out in the temperature range of $100-340 \mathrm{~K}$. The rate of the temperature change was about $1 \mathrm{~K} / \mathrm{min}$. Near the acoustic anomalies, the temperature variation steps were from 1 to $3 \mathrm{~K}$, and at other temperatures, they were $5 \mathrm{~K}$. The temperature dependences of the logarithmic decrement $\delta(T)$ and the resonant frequency $f(T)$ of the composite vibrator were measured. The resonant frequency was used to determine the dynamic Young's modulus $E(T)$ of the samples. The measurements were carried out in the amplitude-independent region at the ultrasonic strain amplitude of $\varepsilon_{0} \sim 1 \cdot 10^{-7}$.

To obtain the information on structure stability of the samples, they were annealed in vacuum at $425 \mathrm{~K}$ for 1 hour.

\section{Results and Discussion}

3.1. Physical Nature of the Dynamic Relaxation. Temperature dependences of the logarithmic decrement $\delta(T)$ measured at frequencies of $f \sim 73 \mathrm{kHz}$ are shown in Figure 1. For comparison, the results obtained earlier for the well-annealed coarse-grained polycrystalline zirconium at the frequency $f \approx 87 \mathrm{kHz}[7,9]$ are also presented. A pronounced peak of internal friction is found at the peak temperature $T_{P} \approx 255 \mathrm{~K}$. 


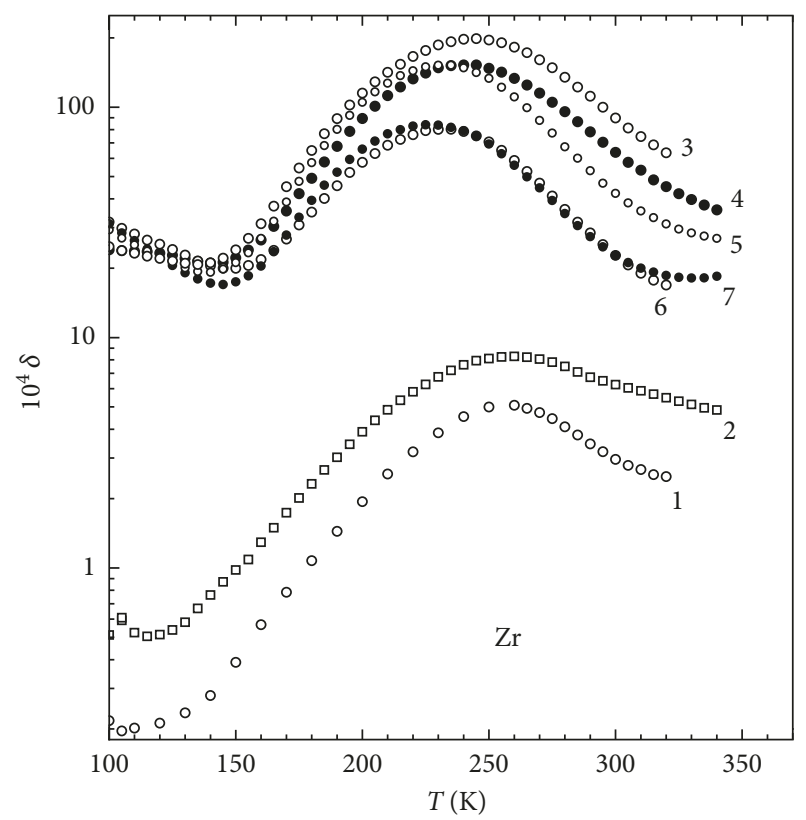

FIgURE 1: Temperature dependences of the logarithmic decrement in zirconium samples with the different mean grain size $\bar{d}$ : $1-\mathrm{Zr}-\mathrm{CG}$, $\bar{d}=150 \mu \mathrm{m} ; 2-\mathrm{Zr}-\mathrm{HEA}, \bar{d}=12 \mu \mathrm{m} ; 3-\mathrm{Zr}-\mathrm{HEAD}, \bar{d}=2 \mu \mathrm{m} ; 4-\mathrm{Zr}-0$, $\bar{d}=392 \mathrm{~nm} ; 5-\mathrm{Zr}-01, \bar{d}=117 \mathrm{~nm} ; 6-\mathrm{Zr}-02 \mathrm{RT}, \bar{d}=113 \mathrm{~nm} ; 7-\mathrm{Zr}-02 \mathrm{CE}$, $\bar{d}=100 \mathrm{~nm}$.

It is established that the peak shifts towards higher temperatures when increasing the frequency, which indicates the thermoactivated relaxation nature of the peak.

In the simplest description of thermally activated dynamic relaxations, it is assumed that all the elementary relaxation entities (relaxators) are identical and, at each temperature, are characterized by an unique relaxation time, whose temperature dependence $\tau(T)$ is described by the Arrhenius expression:

$$
\tau(T)=\tau_{0} \exp \left(\frac{U_{0}}{k T}\right),
$$

where $U_{0}$ is the activation energy of the relaxation, $\tau_{0}$ is the attempt period, and $k$ is the Boltzmann constant. According to the Debye approximation, the frequency-temperature dependence of the relaxation component of the logarithmic decrement $\delta_{\mathrm{r}}^{\mathrm{D}}(\omega, T)$ has the form

$$
\delta_{\mathrm{r}}^{\mathrm{D}}(\omega, T)=\pi \Delta_{\mathrm{M}}^{\mathrm{D}} \frac{\omega \tau(T)}{1+\omega^{2} \tau^{2}(T)},
$$

where $\omega=2 \pi f$ is the angular oscillation frequency, $\Delta_{\mathrm{M}}^{\mathrm{D}}=$ $\left(M_{U}-M_{R}\right) / M_{U}$ is the maximum modulus defect (relaxation strength) associated with the Debye relaxation, and $M_{U}$ and $M_{R}$ are the nonrelaxed and relaxed elastic moduli, respectively. In view of (2), the Debye peak should be observed when the condition

$$
\omega \tau\left(T_{P}^{\mathrm{D}}\right)=1
$$

is satisfied. Notice that the maximum value of the relaxation component of the decrement $\delta_{\mathrm{r} \max }^{\mathrm{D}}$ (the Debye peak height) and the relaxation strength $\Delta_{\mathrm{M}}^{\mathrm{D}}$ are related by

$$
\Delta_{\mathrm{M}}^{\mathrm{D}}=\frac{2}{\pi} \delta_{\mathrm{r} \max }^{\mathrm{D}}
$$

The activation energy $U_{0}$ and the attempt period $\tau_{0}$ can be determined from the peak shift with the frequency change:

$$
\begin{aligned}
& U_{0}=\frac{k}{1 / T_{P}^{\mathrm{D}}\left(\omega_{2}\right)-1 / T_{P}^{\mathrm{D}}\left(\omega_{1}\right)} \ln \frac{\omega_{1}}{\omega_{2}}, \\
& \tau_{0}=\frac{1}{\omega_{1,2}} \exp \left(-\frac{U_{0}}{k T_{P}^{\mathrm{D}}\left(\omega_{1,2}\right)}\right),
\end{aligned}
$$

where $T_{P}^{\mathrm{D}}\left(\omega_{1}\right)$ and $T_{P}^{\mathrm{D}}\left(\omega_{2}\right)$ are the peak temperatures in the temperature dependences measured at the frequencies $\omega_{1}$ and $\omega_{2}$, respectively. The activation energy obtained in this way turned out to be $U_{0} \approx 0.32-0.37 \mathrm{eV}$ and the attempt period $\tau_{0} \sim 10^{-13} \mathrm{~s}$.

The experimentally determined values of the activation parameters allow to attribute the peak to a family of so-called Koiwa-Hasiguti peaks (KH peaks) [10] caused by thermally activated unpinning of dislocations from point defects (impurities and/or vacancies). Then, the parameter $U_{0}$ has the meaning of the binding energy, and the parameter $\tau_{0}$ is the period of oscillations of the microscopic element of the dislocation line directly interacting with the point defect.

The values of $U_{0}$ and $\tau_{0}$ obtained according to the simple Debye approximation ( 5 and 6 ) should be considered only as preliminary estimates. In fact, the frequency-temperature dependence of the relaxation component of the decrement $\delta_{\mathrm{r}}^{\mathrm{KH}}(\omega, T)$ near the $\mathrm{KH}$ peaks is described by a more complicated expression than the Debye peak [10]:

$$
\delta_{\mathrm{r}}^{\mathrm{KH}}=\Delta_{\mathrm{M}}^{\mathrm{KH}} \frac{2}{(\omega \tau)^{-2}+4}\left[1-\exp \left(-\frac{\pi}{\omega \tau}\right)\right],
$$

where $\Delta_{\mathrm{M}}^{\mathrm{KH}}$ is the maximum value of the modulus defect (relaxation strength) associated with the given dynamic relaxation

$$
\Delta_{\mathrm{M}}^{\mathrm{KH}} \approx 2.52 \delta_{\mathrm{r} \max }^{\mathrm{KH}},
$$

with $\delta_{\mathrm{rmax}}^{\mathrm{KH}}$ being the height of the experimentally observed $\mathrm{KH}$ peak. It should be noted that, in this case, the maximum modulus defect $\Delta_{\mathrm{M}}^{\mathrm{KH}}$ is very large in comparison with the peak height $\delta_{\mathrm{r} \max }^{\mathrm{KH}}$ unlike the Debye peak (cf. (4) and (8). It was noted in [10] that the difference between these two relaxations consists in the fact that, in the case of the $\mathrm{KH}$ relaxation, the behavior of pinned dislocations is asymmetric with respect to the application and the release of external stresses. Thus, the solid that contains pinned dislocations as elements of inelastic strain does not behave like standard linear solid for which the Debye approximation is valid. According to [10], in order to observe the $\mathrm{KH}$ peak in the temperature dependence of the decrement, it is necessary that

$$
\omega \tau\left(T_{P}^{\mathrm{KH}}\right)=1.3331,
$$

in contrast to the condition $\omega \tau\left(T_{P}^{\mathrm{D}}\right)=1$ for the Debye relaxation. Within the single relaxation time approximation, the activation energy $U_{0}$ can still be determined from the 
frequency shift of the peak temperature using (5), but for the estimation of the attempt period $\tau_{0}$, (6) should be modified as follows:

$$
\tau_{0}=\frac{1.3331}{\omega_{1,2}} \exp \left(-\frac{U_{0}}{k T_{P}^{\mathrm{KH}}\left(\omega_{1,2}\right)}\right) .
$$

Thus the use of (9) instead of (3) does not affect the evaluation of the activation energy $U_{0}$ and only slightly increases the estimate of the attempt period $\tau_{0}$. This allows us, as before, to consider the thermally activated unpinning of dislocation segments from point defects as the basic microscopic mechanism of this low-temperature relaxation.

3.2. Influence of SPD on the Parameters of the Dynamic Relaxation. As a result of the intensive plastic deformation, the temperature dependences of the decrement $\delta(T)$ underwent a number of essential changes:

(1) Background values of the decrement $\delta_{\mathrm{BG}}(T)$ and the height of the relaxation component of the peak $\delta_{\mathrm{r} \text { max }}^{\mathrm{KH}}=\left(\delta-\delta_{\mathrm{BG}}\right)\left(T_{P}^{\mathrm{KH}}\right)$ increased significantly.

(2) The peak temperature $T_{P}^{\mathrm{KH}}$ systematically decreased.

(3) The peak width $\Delta\left(1 / T_{0.5}^{\mp}\right)^{\mathrm{KH}}=1 / T_{0.5}^{-}-1 / T_{0.5}^{+}$defined as the difference between the inverse temperatures corresponding to the peak half-height level $0.5 \delta_{\mathrm{rmax}}^{\mathrm{KH}}$ slightly decreased.

The changes in the indicated relaxation peak parameters are shown in Figures 2(a)-2(c) and in Table 2. It is assumed that the background losses depend exponentially on temperature [7]:

$$
\delta_{\mathrm{BG}}(T)=A \exp \left(\frac{-B}{k T}\right)+\delta_{\mathrm{BG}}(5 \mathrm{~K}),
$$

where $\delta_{\mathrm{BG}}(5 \mathrm{~K})$ are the background losses at $5 \mathrm{~K}, A$, and $B$ are adjustable parameters.

3.2.1. The Peak Height. Figures 1 and 2(a) show that, at the first stages of SPD application, the peak height increases by more than an order of magnitude compared to undeformed and annealed samples (Figures 1 and 2(a)). Complicating the SPD schemes and increasing the total plastic deformation $e$ (and, correspondingly, decreasing in the mean grain size $\bar{d}$ ) did not lead to a further increase in the height of the relaxation peak. Moreover, there was a tendency to some decrease in peak height for the most fragmented samples.

The height of the relaxation peak $\delta_{\mathrm{r} \text { max }}^{\mathrm{KH}}$ is determined by a number of elementary relaxators being excited in the material and by the individual contribution of each relaxator to inelastic strain of the crystal. The significant increase in the peak height in the deformed samples may indicate a substantial increase in the number of relaxators and/or an increase in their individual contributions. The elementary act of the dynamic $\mathrm{KH}$ relaxation is the thermally activated unpinning of dislocation segments from weak pinning centers (impurities and/or vacancies) under the joint action of the external alternating stress and thermal activation. The significant increase in the dislocation density $\Lambda$ (the total

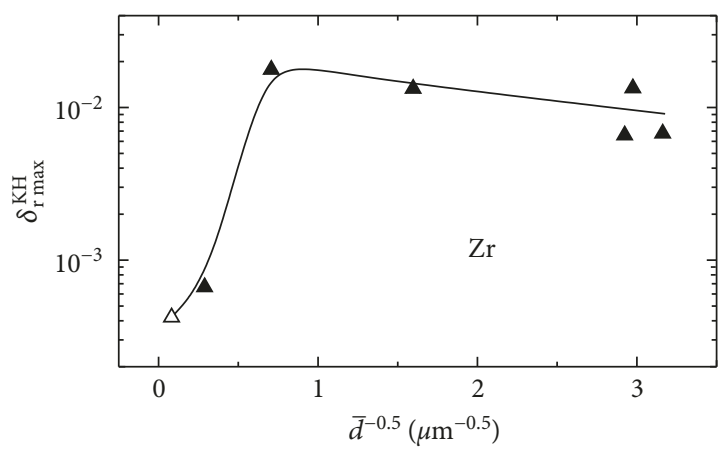

(a)

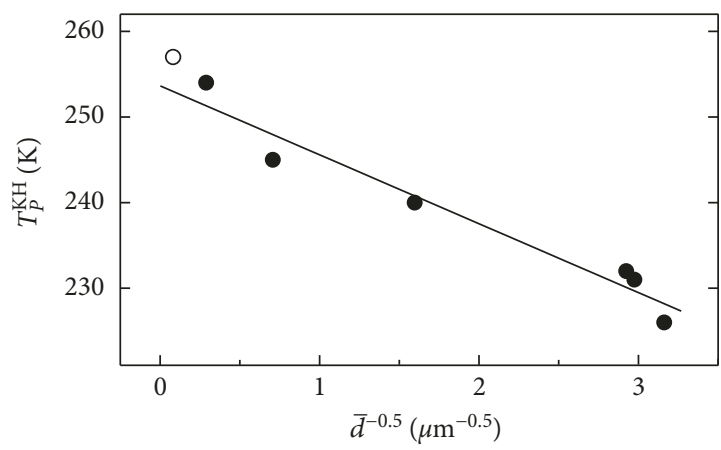

(b)

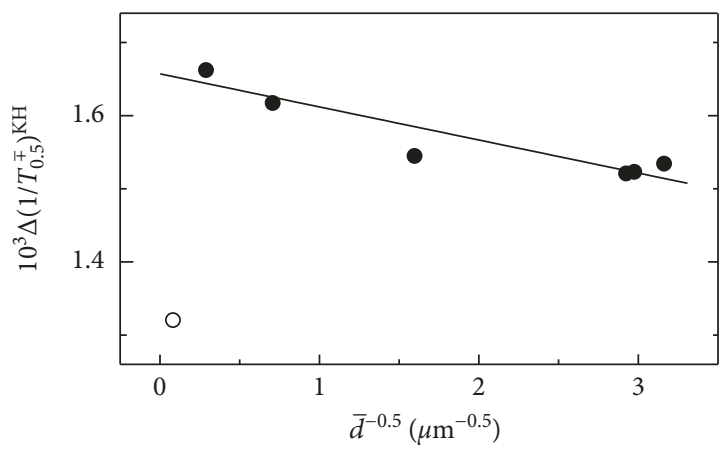

(c)

Figure 2: Dependences of (a) the peak height $\delta_{\mathrm{r} \max }$, (b) the peak temperature $T_{P}$, and (c) the peak width $\Delta 1 / T_{0.5}^{\mp} K H$ in $\mathrm{Zr}$ on the mean grain size $\bar{d}$. Full symbols are the data of this work; empty symbols are taken from [7].

length of dislocation lines in the unit volume) as a result of the SPD may lead to an increase of the number of successful acts of unpinning of dislocation segments from local pinning centers. The contribution of the individual unpinning act to ultrasound absorption is determined by the additional inelastic strain provided by each unpinning. While analysis of the magnitude of this contribution is a rather difficult task even for coarse-grained metals or metallic single crystals [11], it becomes even more complicated in the case of the NS metals obtained by SPD methods. When the value of plastic deformation reaches several hundred percent, all possible modes of plastic deformation act in metals: dislocation sliding, twinning, processes at the grain boundaries are involved, and a dynamic recovery is observed. A complex 
TABLE 2: The main parameters of the $\mathrm{KH}$ peak in the as-prepared samples.

\begin{tabular}{|c|c|c|c|c|c|c|}
\hline Sample & $\bar{d}, \mu \mathrm{m}$ & $T_{P}, \mathrm{~K}$ & $\delta_{\mathrm{r} \max }, \times 10^{-3}$ & $U_{0}, \mathrm{eV}$ & $\Delta\left(1 / \mathrm{T}_{0.5}^{\mp}\right)^{\mathrm{KH}}, 10^{-3} \cdot \mathrm{K}^{-1}$ & $\beta$ \\
\hline Zr-CG & 150 & 257 & 0.421 & 0.34 & 1.321 & 2.52 \\
\hline Zr-HEA & 12 & 254 & 0.666 & 0.37 & 1.662 & 3.82 \\
\hline Zr-HEAD & 2 & 245 & 17.7 & 0.36 & 1.617 & 3.57 \\
\hline $\mathrm{Zr}-0$ & 0.392 & 240 & 13.3 & 0.37 & 1.545 & 3.43 \\
\hline Zr-01 & 0.117 & 232 & 6.58 & 0.35 & 1.521 & 2.97 \\
\hline Zr-02RT & 0.113 & 231 & 13.4 & 0.36 & 1.523 & 3.12 \\
\hline $\mathrm{Zr}-02 \mathrm{CE}$ & 0.100 & 226 & 6.76 & 0.32 & 1.534 & 2.88 \\
\hline
\end{tabular}

$\bar{d}$ is the mean grain size; $T_{P}$ is the peak temperature; $\delta$ is the measured value of the decrement; $\delta_{\mathrm{r} \max }=\left(\delta-\delta_{\mathrm{BG}}\right)_{\max } ; \delta_{\mathrm{BG}}$ is the background decrement; $U_{0}$ is the activation energy; $\Delta\left(1 / \mathrm{T}_{0.5}^{\mp}\right)^{\mathrm{KH}}$ is the peak width; $\beta$ is the parameter of the relaxation time distribution.

subsystem of crystal defects with a high level of internal stresses is formed in the samples in this case. The dislocation density reaches values of $\Lambda \sim 10^{14}-10^{15} \mathrm{~m}^{-2}$. Along with the density of intragranular, the density of grain-boundary dislocations considerably increases. The situation in HCP metals is even more complicated in view of the presence of essentially different slip systems: basic, prismatic, and two pyramidal [12]. Under these conditions, it is difficult to assess the degree of influence of SPD on the contribution of the individual relaxator to the ultrasound absorption. We can only state a significant increase in the total relaxation strength as a result of the application of the SPD schemes chosen. It should be emphasized that, even in the most fragmented samples with the mean grain size of about $100 \mathrm{~nm}$, there is a sufficiently large number of dislocation segments capable of thermally activated unpinning from local pinning centers and contributing to the dynamic $\mathrm{KH}$ relaxation.

It should be noted that a use of SPD does not always lead to an increase in the background absorption and the relaxation strength. In the work [13], some lowering of the damping capacity was observed at a comparative study of coarse-grained and ECAP-refined UFG samples of $\mathrm{Fe}-$ $13 \mathrm{Cr}-2 \mathrm{Al}-1 \mathrm{Si}$ ferromagnetic alloy. This was attributed to the pinning effect introduced by SPD crystal defects (dislocations, subgrain boundaries, etc.) on the movement of magnetic domain walls.

3.2.2. The Peak Temperature. The most interesting effects that have been registered in this work are the systematic lowering of the peak temperature $T_{P}^{\mathrm{KH}}$ and the decrease of the peak width $\Delta\left(1 / \mathrm{T}_{0.5}^{\mp}\right)^{\mathrm{KH}}$ (peak narrowing) with the decrease of the mean grain size in the samples (Figures 2(b) and 2(c) and Table 2). Combining (1) and (9), the KH peak position along the temperature axis can be determined:

$$
T_{P}^{\mathrm{KH}}=\frac{U_{0}}{k \ln \left(1.3331 / \omega \tau_{0}\right)}
$$

Equation (12) shows that, at a constant oscillation frequency $\omega$ (i.e., almost the case in our experiment), the peak shifts towards low temperatures as a result of the SPD can be attributed to a decrease in both the activation energy $U_{0}$ and the attempt period $\tau_{0}$. It should be noted that the dependence of $T_{P}^{\mathrm{KH}}$ on $\tau_{0}$ is logarithmic and is much weaker. Some additional experimental evidences and arguments are required in order to make the final choice between these two possible factors affecting the peak temperature (see below).

3.2.3. The Peak Width. As it was noted, the width of the experimentally observed $\mathrm{KH}$ peak $\Delta\left(1 / \mathrm{T}_{0.5}^{\mp}\right)^{\mathrm{KH}}$ decreases systematically with decreasing mean grain size in the samples. The value of $\Delta\left(1 / \mathrm{T}_{0.5}^{\mp}\right)^{\mathrm{KH}}$ in the single relaxation time approximation can be obtained by equating the right-hand side of (7) to $0.5 \delta_{\mathrm{r} \text { max }}^{\mathrm{KH}}(8)$ and finding roots of the equation

$$
\frac{10.08}{y^{-2}+4}\left[1-\exp \left(\frac{-\pi}{y}\right)\right]-1=0,
$$

where $y_{1,2} \equiv(\omega \tau)_{1,2}$ are the values of $\omega \tau$ at which the half height $0.5 \delta_{\mathrm{rmax}}^{\mathrm{KH}}$ of the peak is reached. The numerical solution of (13) shows that the half height of the $\mathrm{KH}$ peak is reached at $\omega \tau_{1}=6.178$ and $\omega \tau_{2}=0.405$. Assuming that the values of $\omega, U_{0}$, and $\tau_{0}$ in the interval between $\omega \tau_{1}$ to $\omega \tau_{2}$ remain unchanged, it is easy to obtain

$$
\Delta\left(\frac{1}{T_{0.5}^{\mp}}\right)^{\mathrm{KH}}=2.725 \frac{k}{U_{0}} .
$$

The analogous expression for the width of the Debye peak can be obtained analytically under the same assumptions and that differs from (14) only by a numerical coefficient [14]:

$$
\Delta\left(\frac{1}{T_{0.5}^{\mp}}\right)^{D}=2.634 \frac{k}{U_{0}} .
$$

It follows from (14) and (15) that the widths of the relaxation peaks of both types are inversely proportional to the activation energy $U_{0}$. According to (12), the peak temperature $T_{P}^{\mathrm{KH}}$ is directly proportional to this value. In our experiments, both the peak temperature and peak width decreased with decreasing the mean grain size. It is clear that these effects cannot be explained simultaneously by a change (decreasing or increasing) of $U_{0}$. On the other hand, according to (15), the peak width does not depend on $\tau_{0}$. This allows us to suppose that a decrease in $\tau_{0}$ may be the reason of both effects.

3.2.4. Relaxation Time Distribution. The registered changes in the parameters of the $\mathrm{KH}$ peak due to SPD are apparently caused by changes in the effective values of the activation 
parameters of the $\mathrm{KH}$ relaxation process. The important consequence of SPD is the appearance of significant internal stresses in the metal which may either facilitate or hinder unpinning of dislocation segments from the pinning centers thus changing the effective values of the activation parameters $U_{0}$ and/or $\tau_{0}$ and, hence, the relaxation time $\tau$. These changes may be different for relaxators acting at different locations in the sample. This should lead to the formation of spectra (discrete or continuous) of the activation parameters. The large width of the relaxation peaks may serve as an evidence of the existence of the relaxation time spectra. The temperature dependences of the normalized relaxation components of the decrement $\delta_{\mathrm{r}}^{\mathrm{KH}} / \delta_{\mathrm{r} \max }^{\mathrm{KH}}$ for three samples are presented in Figure 3. The dashed curve is drawn using (7) for the case of a single relaxation time approximation. It is clearly seen that the experimentally measured internal friction peaks are much broader than that predicted by (7).

In the literature, several ways of taking into account the distribution of the relaxation time were proposed for describing the broadened relaxation peaks. The use of the normal Gaussian distribution for the value of $\ln (\tau)$ (lognormal distribution) we consider is most reasonable $[15,16]$. In [15], such analysis was used when investigating the influence of the relaxation time distribution on the width and height of the Debye peaks. In this paper, we applied the same approach to the analysis of properties of the $\mathrm{KH}$ peaks.

The relaxation component of the logarithmic decrement when considering the distribution in $\ln (\tau)$ can be written as

$\delta_{\mathrm{r}}^{\mathrm{KH}}=\Delta_{\mathrm{M}}^{\mathrm{KH}} \int_{-\infty}^{+\infty} \Psi(\ln (\tau)) \frac{2}{(\omega \tau)^{-2}+4}\left[1-\exp \left(-\frac{\pi}{\omega \tau}\right)\right] d(\ln (\tau))$,

where $\Psi(\ln (\tau)) d(\ln (\tau))$ is the relative number of elementary processes contributing to $\delta_{\mathrm{r}}^{\mathrm{KH}}$, for which the logarithm of the relaxation time falls within the interval between $\ln (\tau)$ and $\ln (\tau)+d(\ln (\tau))$. The function $\Psi(\ln \tau)$ is a normalized distribution function:

$$
\int_{-\infty}^{+\infty} \Psi(\ln (\tau)) d(\ln (\tau))=1
$$

Let us change the absolute value of $\tau$ to the normalized one and introduce the variable

$$
z=\ln \left(\frac{\tau}{\tau_{m}}\right)
$$

where $\tau_{m}$ is the mean value of the relaxation time at the given temperature. Then the Gaussian distribution function for $z$ can be written as

$$
\Psi(z)=\frac{1}{\beta \sqrt{\pi}} \exp \left[-\left(\frac{z}{\beta}\right)^{2}\right]
$$

where $\beta$ is the Gaussian distribution parameter, which determines the half-width of the distribution at the level $\Psi(z) / \Psi\left(z_{m}\right)=1 / e, \Psi\left(z_{m}\right)$ is the maximum value of $\Psi$, and $z_{m} \equiv z\left(\tau_{m}\right)=0$ is the average of distribution. Substituting (19) in (16) and introducing variables

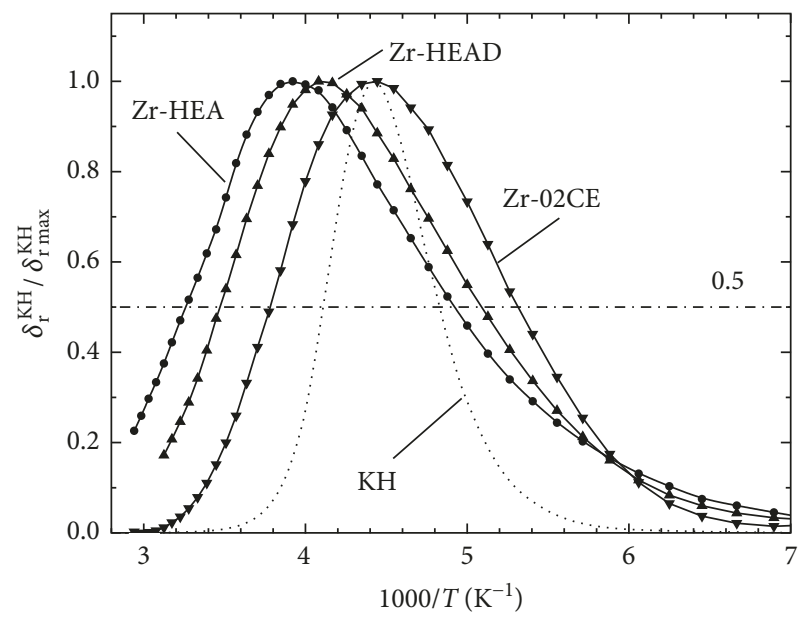

FIgURE 3: The normalized dependences of the relaxation components of the decrement on the inverse temperature $\delta_{\mathrm{r}}^{\mathrm{KH}} / \delta_{\mathrm{r} \text { max }}^{\mathrm{KH}}(1 / T)$ (for better clarity, the data for three samples are given). The dashed line shows the normalized dependence of the relaxation component of the decrement obtained for the sample $\mathrm{Zr}$-02CE in the single relaxation time approximation (7).

$$
\begin{gathered}
x=\ln \frac{\omega \tau_{m}}{1.3331}, \\
u=\frac{z}{\beta},
\end{gathered}
$$

one obtains the expression for the relaxation component of the decrement taking into account the Gaussian distribution in $\ln \left(\tau / \tau_{m}\right)$ :

$$
\delta_{\mathrm{r}}^{\mathrm{KH}}=\Delta_{\mathrm{M}}^{\mathrm{KH}} f_{2}^{\mathrm{KH}}(x, \beta)
$$

where

$$
\begin{array}{r}
f_{2}^{\mathrm{KH}}(x, \beta)=\frac{2}{\sqrt{\pi}} \int_{-\infty}^{+\infty} \exp \left(-u^{2}\right) \\
\times\left\{1-\exp \left[-\frac{\pi}{1.3331 \cdot \exp (x+\beta u)}\right]\right\} \\
\times \frac{d u}{4+(1.3331)^{-2} \cdot \exp [-2(x+\beta u)]} .
\end{array}
$$

This latter expression uses the notation introduced in [15]. The quantity $\beta$ in (23) determines the change of the main characteristics of the $\mathrm{KH}$ peak in the presence of the relaxation time distribution. For $\beta=0$, the Gaussian function goes into a Dirac $\delta$-function and (23) degenerates into (7) for the case of a single relaxation time. In Figure 4, the normalized curves for values of $\beta=0,2,5$, and 10 are presented.

An analysis of (23) allows us to draw some conclusions about the changes of the basic parameters of the $\mathrm{KH}$ peak in the presence of the distribution of relaxation times (in other 


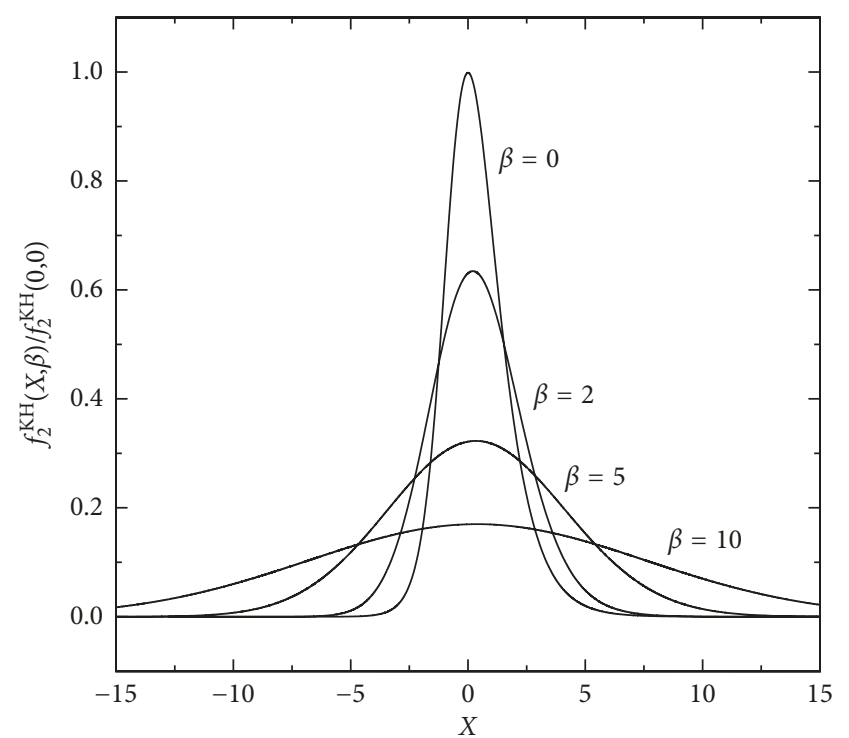

FIGURE 4: The influence of the distribution parameter $\beta$ on the width and height of the $\mathrm{KH}$ peak (Equation 23).

words, about their dependence on the distribution parameter $\beta$ ). First of all, a considerable increase in the width of the relaxation peak with increasing $\beta$ is observed. It was demonstrated in [15] that the ratio

$$
r_{2}^{\mathrm{KH}}=\frac{\Delta\left(1 / T_{0.5}^{\mp}\right)^{\mathrm{KH}}(\beta)}{\Delta\left(1 / T_{0.5}^{\mp}\right)^{\mathrm{KH}}(0)}
$$

is a useful characteristic of the peak width (here $\Delta\left(1 / \mathrm{T}_{0.5}^{\mp}\right)^{\mathrm{KH}}(\beta)$ and $\Delta\left(1 / \mathrm{T}_{0.5}^{\mp}\right)^{\mathrm{KH}}(0)$ are the widths of the peaks with $\beta>0$ and $\beta=0$, resp.). The value $\Delta\left(1 / \mathrm{T}_{0.5}^{\mp}\right)^{\mathrm{KH}}(0)$ can be obtained using (14). Numerical calculations give an empirical relation between the values $r_{2}$ and $\beta$. In Figure 5, the dependences of $\beta^{\mathrm{KH}}\left(r_{2}\right)$ for the $\mathrm{KH}$ peak and $\beta^{\mathrm{D}}\left(r_{2}\right)$ for the Debye peak are presented. Solid lines show the corresponding approximations for $\beta^{\mathrm{KH}}$ and $\beta^{\mathrm{D}}$

$$
\begin{aligned}
& \beta^{\mathrm{KH}}=\left(r_{2}-1\right)^{0.276}+1.618\left(r_{2}-1\right), \\
& \beta^{D}=\left(r_{2}-1\right)^{0.366}+1.493\left(r_{2}-1\right) .
\end{aligned}
$$

These approximations can be used for obtaining the distribution parameter $\beta$ from the experimental data by calculating $r_{2}$ in accordance with (14), (15), and (24). Figure 6(a) shows the dependences of the experimentally measured $(\beta>0)$ and calculated, according to (14) $(\beta=0)$, KH peak widths $\Delta\left(1 / \mathrm{T}_{0.5}^{\mp}\right)^{\mathrm{KH}}$ on the mean grain size $\bar{d}$. Figure $6(\mathrm{~b})$ shows a change of the distribution parameter $\beta$ when decreasing the mean grain size $\bar{d}$.

Analysis of (23) shows that, along with broadening of the $\mathrm{KH}$ peak with increasing $\beta$, a significant decrease in the height of its relaxation component $f_{2}^{\mathrm{KH}}(0, \beta)$ takes place (Figure $7(\mathrm{a})$ ). The relationship $f_{2}^{\mathrm{KH}}(0, \beta)$ may be approximated by the expression

$$
f_{2}^{\mathrm{KH}}(0, \beta)=0.03+\frac{0.37}{1+0.21 \beta^{1.65}} .
$$

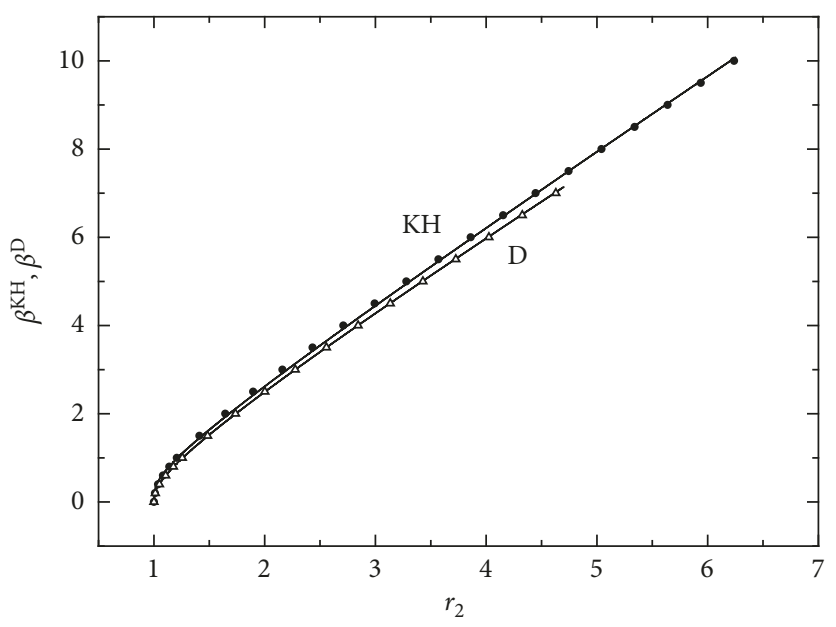

FIGURE 5: The relationship between the value of $r_{2}$ and the distribution parameters for the Koiwa-Hasiguti peaks $\beta^{\mathrm{KH}}$ (the present work) and for the Debye peaks $\beta^{\mathrm{D}}$ (the data from [14] were processed). The points correspond to numerical calculations; the solid lines show the approximations $\beta^{\mathrm{KH}}\left(r_{2}\right)$ and $\beta^{\mathrm{D}}\left(r_{2}\right)((25)$ and (26), resp.).

The latter approximation is shown in Figure 7 (a) by a solid line. The relaxation strength $\Delta_{\mathrm{M}}^{\mathrm{KH}}$ can be derived from the height of the relaxation peak $\delta_{\mathrm{rmax}}^{\mathrm{KH}}$

$$
\Delta_{\mathrm{M}}^{\mathrm{KH}}=\frac{\delta_{\mathrm{r} \max }^{\mathrm{KH}}}{f_{2}^{\mathrm{KH}}(0, \beta)} \text {. }
$$

The value $\Delta_{\mathrm{M}}^{\mathrm{KH}}$ can also be obtained independently from the measurements of the elastic moduli which are usually carried out together with the internal friction measurements

$$
\Delta_{\mathrm{M}}^{\mathrm{KH}}=\frac{E_{\mathrm{U}}^{\mathrm{KH}}-E_{\mathrm{R}}^{\mathrm{KH}}}{E_{\mathrm{U}}^{\mathrm{KH}}},
$$

where $E_{\mathrm{U}}^{\mathrm{KH}}$ and $E_{\mathrm{R}}^{\mathrm{KH}}$ are the values of the unrelaxed and relaxed elastic moduli, respectively. The applicability of this method is limited by the correct determination of $E_{\mathrm{U}}^{\mathrm{KH}}$ and $E_{\mathrm{R}}^{\mathrm{KH}}$ from the experiment. The value of $E_{\mathrm{R}}^{\mathrm{KH}}$ should be taken in the temperature range where all the relaxators of the given type already contribute to the modulus defect (a hightemperature limit). Likewise, it is not easy to obtain the value of $E_{\mathrm{U}}^{\mathrm{KH}}$ since it assumes that, in the same specimen and in the same temperature range, all the relaxators of this type are completely immobilized (or absent) and do not contribute to the modulus defect at all. In most of the cases, it is impossible to fulfill simultaneously these concurrent conditions, first of all, due to superposition of two (or several) relaxation processes with close activation parameters. Usually, the value of $E_{\mathrm{R}}^{\mathrm{KH}}$ is taken in the temperature range $T \gg T_{P}$. When studying relaxation processes initiated by plastic deformation, the values of elastic moduli in wellannealed samples are usually taken as values of $E_{\mathrm{U}}^{\mathrm{KH}}$. With such a choice of $E_{\mathrm{U}}^{\mathrm{KH}}$ and $E_{\mathrm{R}}^{\mathrm{KH}}$, the risk of underestimation of $\Delta_{\mathrm{M}}^{\mathrm{KH}}$ remains quite high.

In Figure 8 , the dependences of $\Delta_{\mathrm{M}}^{\mathrm{KH}}$ on the mean grain size $\bar{d}$ obtained by both methods are shown. As the 


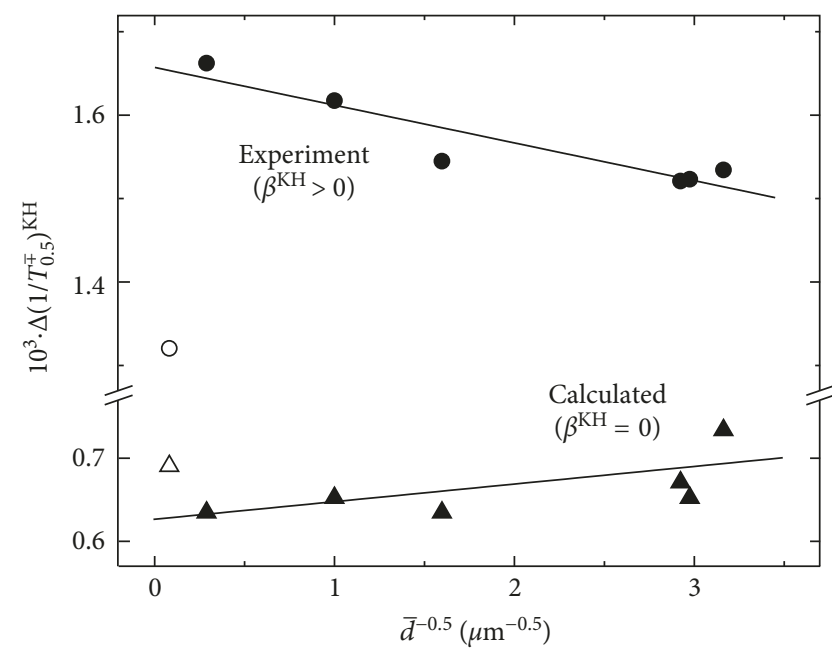

(a)

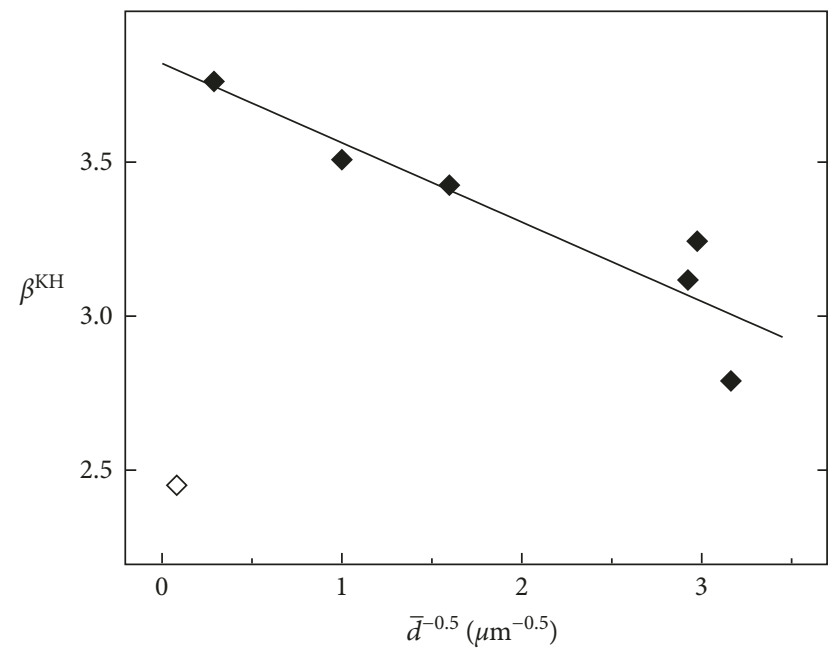

(b)

FIGURE 6: Dependences of (a) the experimentally measured width $\Delta 1 / T_{0.5}^{\mp}{ }^{K H}$ of the $\mathrm{KH}$ peak and calculated according to (14) for the case $\beta^{\mathrm{KH}}=0$ and (b) the distribution parameter $\beta^{\mathrm{KH}}$ on the mean grain size $\bar{d}$. Full symbols are the data of the present work; open symbols are the processed data of the work [7].

unrelaxed $E_{\mathrm{U}}^{\mathrm{KH}}(T)$, the temperature dependence $E(T)$ in the well-annealed Zr-CG sample [17] was used in (29). Qualitatively, the dependences obtained by two independent methods correlate with each other, although the values $\Delta_{\mathrm{M}}^{\mathrm{KH}}$ obtained from the measurements of the dynamic Young's modulus appeared to be smaller than those obtained from the peak height. It should be noted that, despite the underestimation, the values $\Delta_{\mathrm{M}}^{\mathrm{KH}}$ obtained from the Young's modulus measurements are more than twice (from 2.2 to 2.8 times) higher than the peak height $\delta_{\mathrm{r} \max }^{\mathrm{KH}}$. The significant magnitude of the modulus defect is an additional evidence of the fact that this peak is the $\mathrm{KH}$ peak [10].

Figure 8, as well as Figure 2(a), shows that the relaxation strength of the $\mathrm{KH}$ relaxation decreases with increasing a degree of fragmentation of the samples. This means that, in the most fragmented samples, the total number of successful

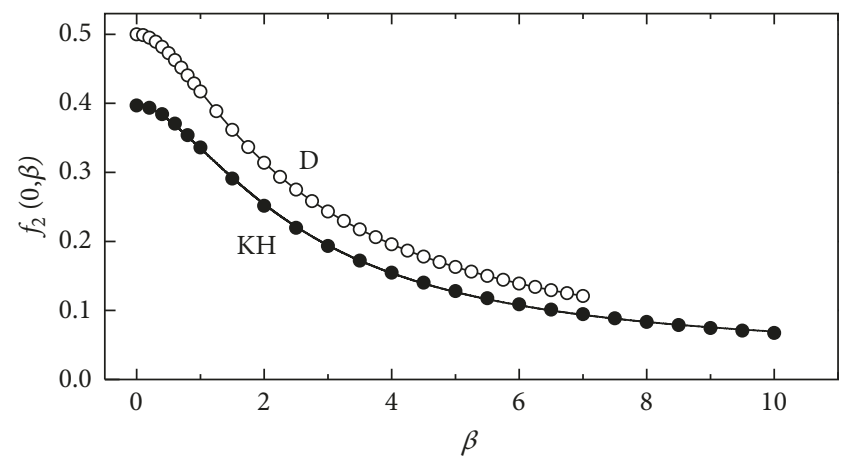

(a)

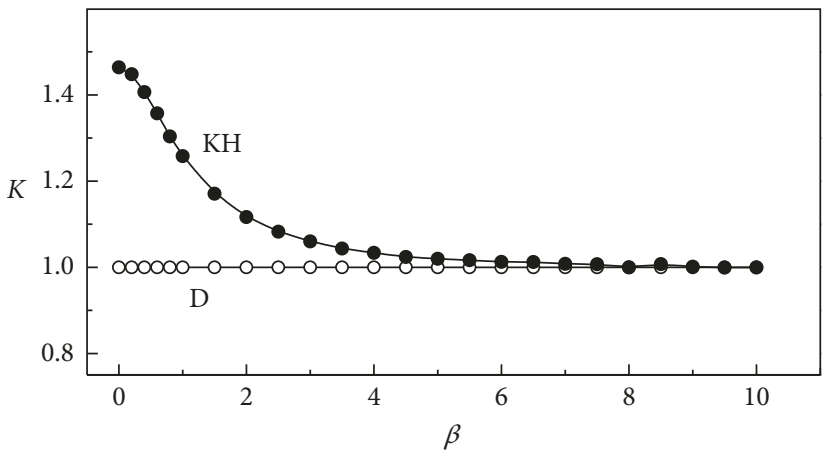

(b)

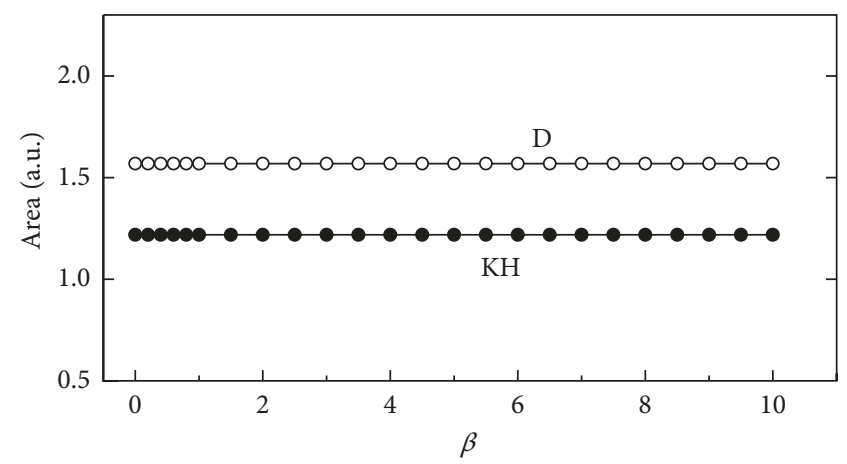

(c)

FIGURE 7: Dependences on the parameter $\beta$ of (a) the height of the relaxation component $f_{2}^{\mathrm{KH}}(0, \beta)$ of the $\mathrm{KH}$ peak (the solid line is the approximation by (27)), (b) the ratios $K$ of the derivatives at the inflection points on the left and right sides of the $\mathrm{KH}$ peak (30), and (c) the area $\int_{-\infty}^{\infty} f_{2} x, \beta d x$ under the peak. Filled symbols refer to the $\mathrm{KH}$ peak; open symbols refer to the Debye peak.

acts of thermally activated unpinning of dislocations from the pinning centers gradually decreases that can be explained by a decrease in the mean length of dislocation segments $L_{c}$ with a decrease in the mean grain size in submicrocrystalline samples.

3.2.5. The Shape of the Peak. A more detailed analysis of the behavior of the $\mathrm{KH}$ peak in the presence of the relaxation time distribution shows that an increase in $\beta$ significantly changes the shape of the $\mathrm{KH}$ peak. Contrary to the Debye peak, the $\mathrm{KH}$ 


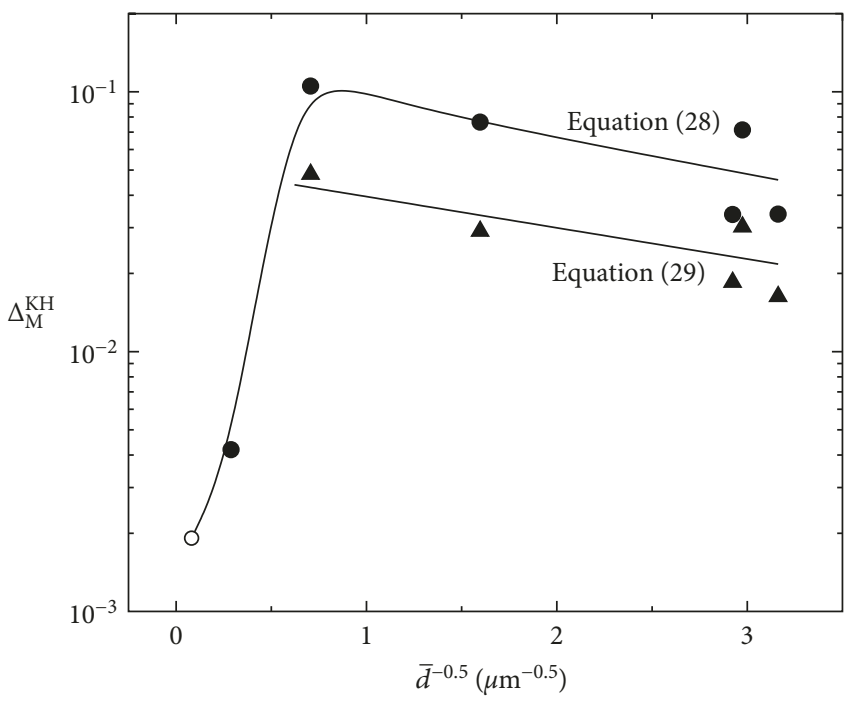

FIgURE 8: Dependences of the relaxation strength $\Delta_{\mathrm{M}}^{\mathrm{KH}}$ on the mean grain size $\bar{d}$ obtained from the $\mathrm{KH}$ peak height (Equation 28) and from the measurements of the dynamic Young's modulus (29).

peak at $\beta=0$ is asymmetric. The slope of its high-temperature branch is noticeably steeper than that of the low-temperature one. As a measure of the peak asymmetry, the absolute value of the ratio $K$ of the derivatives at the inflection points on both sides of the peak may be used [19]:

$$
K=\left|\frac{\left.(d / d x) f_{2}(x, \beta)\right|_{\max }}{\left.(d / d x) f_{2}(x, \beta)\right|_{\min }}\right| .
$$

While the Debye peak in the coordinates chosen is symmetric with respect to $x=0$ and $\mathrm{K}^{\mathrm{D}}=1$ regardless of the value of $\beta$, the $\mathrm{KH}$ peak at $\beta=0$ has a noticeable asymmetry and $\mathrm{K}^{\mathrm{KH}}=1.464$. When $\beta$ increases, the quantity $\mathrm{K}^{\mathrm{KH}}$ decreases rather rapidly and tends to unity in the limit of large $\beta$; that is, the $\mathrm{KH}$ peak becomes almost symmetrical (Figure $7(\mathrm{~b})$ ). This result does not agree with the conclusion made in [19] where it is stated that the shape of the $\mathrm{KH}$ peak remains unchanged (i.e., $K=$ const), and the peak retains its asymmetry when increasing a dispersion of the activation energy.

Despite the changes in the width, height, and shape of the KH peak, the area under the peak $\int_{-\infty}^{\infty} f_{2}^{\mathrm{KH}}(x, \beta) d x$ does not depend on the distribution parameter and remains unchanged for any $\beta$ (Figure $7(\mathrm{c})$ ). This result agrees with the analogous result obtained in [15] for the Debye peak.

3.3. Effect of Annealing. To test the stability of the structures created in the samples during SPD and to detect a possible recovery of acoustic properties, the samples were annealed at the temperature $T_{\mathrm{ann}}=425 \mathrm{~K}$ for 1 hour in vacuum. The annealing temperature was chosen close to the lower boundary of the primary recrystallization temperature $T_{\text {cryst }} \approx 0.2 T_{m}$ ( $T_{m}=2098 \mathrm{~K}$ is the melting point of zirconium).

It is well known that significant changes can occur in the structure of the samples during annealing. Even at temperatures under $T_{\text {cryst }}$, the parameters of the dislocation structure begin to change; in particular, the dislocation density $\Lambda$ decreases and the mean length of dislocation segments $L_{c}$ changes. When $T_{\text {cryst }}$ is reached, growth of small grains is occurred, and the mean grain size increases. That may be accompanied by a formation of annealing textures (a predominant growth of grains of certain orientations is observed). Moreover, significant changes of the internal stresses can occur at elevated temperatures due to appearance of additional channels for their relaxation and the increase in the rate of diffusion processes. All these changes should affect the main parameters of the internal friction peaks observed.

3.3.1. Changes in the Peak Temperature. The peak temperature $T_{P}^{\mathrm{KH}}$ increased after annealing; that is, a partial recovery of this $\mathrm{KH}$ peak parameter is observed (Table 3 ). The largest recovery is observed in the most fragmented samples. It means that the structures of the NS samples created with the application of more complicated SPD schemes proved to be the least resistant to the post-SPD heat treatment. According to (12), the value $T_{P}^{\mathrm{KH}}$ is determined by a combination of values $U_{0}$ and $\tau_{0}$. Hence, the observed recovery of $T_{P}^{\mathrm{KH}}$ as the result of the recrystallization may be regarded as evidence of an influence (direct or indirect) of the average grain size on the activation parameters of the $\mathrm{KH}$ relaxation. However, the nature of such influence still remains unclear, and a further study of this problem is required.

3.3.2. Changes in the Peak Width. Unlike the peak temperature, the peak width (or the distribution parameter $\beta$ ) did not undergo a recovery upon annealing and continued to decrease (Table 3). It indicates that, at least at the initial stage of recrystallization, a scatter of the activation parameters of the dynamic $\mathrm{KH}$ relaxation does not increase and even somewhat decreases with increasing fragmentation of the samples. Obviously, the reason for this decrease is different from that leading to a decrease of $\beta$ due to fragmentation of the samples. While the decrease in $\beta$ when decreasing the average grain size $\bar{d}$ may be associated with a narrowing the dislocation segment length distribution at the expense of the longest segments with the lengths $L>\bar{d}$, the decrease in $\beta$ during annealing may indicate further homogenization of this distribution due to a decrease in the dislocation density and a partial relaxation of internal stresses.

3.3.3. Changes in the Relaxation Strength. The values of the relaxation strength obtained both from the peak height and from the dynamic Young's modulus measurements decrease after annealing in all the samples (28, 29, and Table 3); that is, this parameter of the $\mathrm{KH}$ relaxation, like the peak temperature, has a tendency to recovery. The decrease in the relaxation strength is apparently due to a decrease of the number of successful elementary relaxation acts as a consequence of the decrease in the total dislocation density.

3.4. Theory versus Experiment. Concluding the discussion of the results on the dynamic $\mathrm{KH}$ relaxation in submicrocrystalline 
TABLE 3: Change of the $\mathrm{KH}$ peaks parameters after annealing for 1 hour at $425 \mathrm{~K}$.

\begin{tabular}{lcccccccc}
\hline \multirow{2}{*}{ Sample } & \multicolumn{2}{c}{ Zr-0 } & \multicolumn{2}{c}{ Zr-01 } & \multicolumn{2}{c}{ Zr-02RT } & \multicolumn{2}{c}{ Zr-02CE } \\
& Before & After & Before & After & Before & After & Before & After \\
\hline$T_{P}, \mathrm{~K}$ & 240 & $242(+2)$ & 232 & $236(+4)$ & 231 & $239(+8)$ & 226 & $236(+10)$ \\
$\beta$ & 3.43 & $3.42(-0.01)$ & 2.97 & $2.65(-0.32)$ & 3.12 & $2.6(-0.52)$ & 2.88 & $2.3(-0.58)$ \\
$10^{2} \cdot \Delta_{\mathrm{M}}^{\mathrm{KH}},(28)$ & 7.64 & $5.33(-30.2 \%)$ & 3.37 & $2.49(-26.1 \%)$ & 7.13 & $6.35(-10.9 \%)$ & 3.38 & $2.69(-20.4 \%)$ \\
$10^{2} \cdot \Delta_{\mathrm{M}}^{\mathrm{KH}},(29)$ & 2.91 & $2.54(-12.7 \%)$ & 1.85 & $1.60(-13.5 \%)$ & 3.02 & $2.56(-16.2 \%)$ & 1.63 & $1.08(-33.7 \%)$ \\
\hline
\end{tabular}

The changes in the corresponding values as a result of annealing are indicated in parentheses.

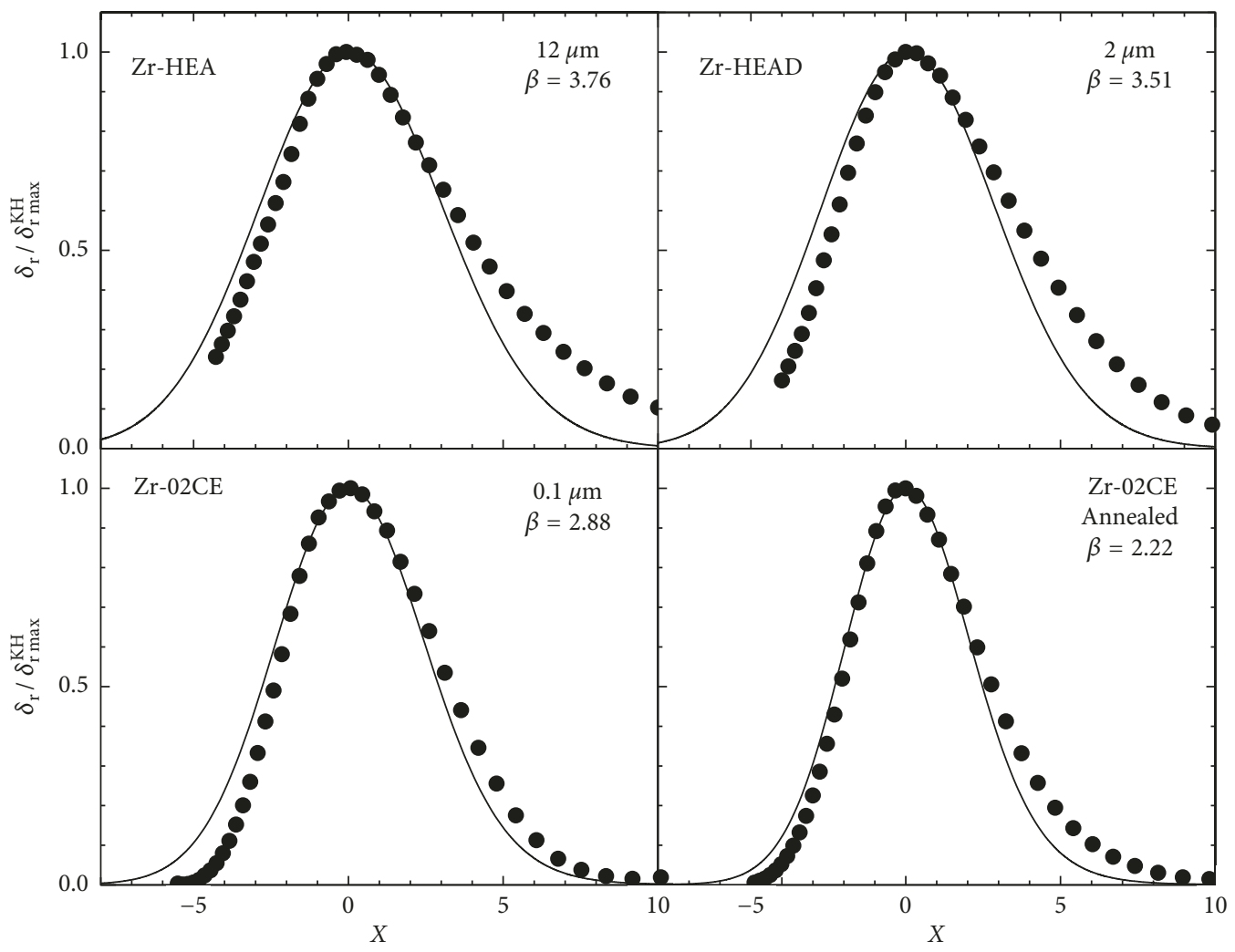

Figure 9: Experimental (points) and theoretical (solid lines) dependences of the normalized relaxation components of the decrement in the samples shown in Figure 3. The variable $x=\left(U_{0} / k\right)\left(1 / T-1 / T_{P}\right)$ is chosen as the abscissa axis for the experimental curves (31); the values of $U_{0}$ and $T_{P}$ are taken from Table 2 .

zirconium, we provide some examples showing that the normalized theoretical dependence $f_{2}^{\mathrm{KH}}(x, \beta) / f_{2}^{\mathrm{KH}}(0, \beta)$ agrees rather well with the experimental dependences $\delta_{\mathrm{r}}^{\mathrm{KH}}(x, \beta) / \delta_{\mathrm{r} \text { max }}^{\mathrm{KH}}(0, \beta)$ obtained on different samples, both in the deformed and annealed states (Figure 9). To perform this procedure, another variable was chosen as the abscissa axis [20] instead of the variable $x$ given by (20):

$$
x=\frac{U_{0}}{k}\left(\frac{1}{T}-\frac{1}{T_{P}}\right) .
$$

Equation (20) can be used in the analysis of the experimental data obtained at a constant temperature (i.e., when $\tau_{m}=$ const) and changing oscillation frequency $f=\omega / 2 \pi$. However, in our experiment, another limiting case was realized: at practically constant oscillation frequency ( $f \approx$ const), the temperature $T$ was varied. Correspondingly, the relaxation time $\tau_{m}$ varied exponentially with $T$ within a rather wide range.
The values of $U_{0}$ and $T_{P}$ in (31) were taken from Table 2 . Values $\beta^{\mathrm{KH}}$ were obtained according to (14), (24), and (26) and data from Table 2. Equation (31) is valid under the assumption that the activation parameters and $\beta^{\mathrm{KH}}$ of the given relaxation process do not depend substantially on temperature.

Figure 9 shows a satisfactory agreement between the theoretical and experimental curves, in particular, in what concerns the width of the peak. It should be noted, however, that there is a significant asymmetry in the experimentally observed peaks, which is larger than that predicted by (30). This could be caused by various reasons. The main of them is the presence of other relaxation processes in $\mathrm{Zr}$ in the temperature range $T<T_{P}^{\mathrm{KH}}$ (see $[6,18]$ for more details) which deform the low-temperature branch of the $\mathrm{KH}$ peak.

In addition to the distortion of the peak shape, the lowtemperature relaxation processes of a different nature may lead to an overestimation of the values $\Delta\left(1 / \mathrm{T}_{0.5}^{\mp}\right)^{\mathrm{KH}}, r_{2}^{\mathrm{KH}}$, 
$\beta^{\mathrm{KH}}$, and $\Delta_{\mathrm{M}}^{\mathrm{KH}}$ determined from the height of the peak. We established that annealing leads to a substantial decrease of the contribution of the low-temperature relaxation processes. Apparently, this can explain the fact that, in the annealed samples, the best agreement between the theoretical and experimental dependences is observed (Figure 9).

\section{Conclusions}

In this work, the detailed experimental study of influence of severe plastic deformation (SPD) and post-SPD heat treatments on the parameters of the low-temperature Koiwa-Hasiguti dynamic relaxation in coarse-grained and nanostructured $\mathrm{Zr}$ samples is carried out. Experimental data on changing the relaxation peak temperature $T_{P}^{\mathrm{KH}}$, the relaxation strength $\Delta_{\mathrm{M}}^{\mathrm{KH}}$, and the relaxation time distribution parameter $\beta^{\mathrm{KH}}$ with the mean grain size $\bar{d}$ are obtained. The analysis of our results allows conclude the following.

(1) As a result of SPD, the sound absorption in all samples increases significantly both the background decrement $\delta_{\mathrm{BG}}(T)$ and the values of the KH peak height $\delta_{\mathrm{r} \max }^{\mathrm{KH}}$. This effect is due to a significant increase in the dislocation density in samples during the SPD processes. The largest increase in the decrement is observed at the first stages of SPD. With further accumulation of the total plastic deformation and decreasing the mean grain size, a tendency to a certain decrease in the peak height is observed, which may be caused by decreasing the probability of dislocation unpinning owing to the significant fragmentation of the grain structure.

(2) The experimentally measured peak width $\Delta\left(1 / \mathrm{T}_{0.5}^{\mp}\right)^{\mathrm{KH}}$ turned out to be much larger than that predicted by the theoretical consideration of this relaxation under the assumption of a single relaxation time for all the relaxators. The broadening of the peak is due to a dispersion of $\tau$ around a mean value of the relaxation time $\tau_{m}(T)$. Based on the lognormal distribution of the value $\ln (\tau)$, the statistical analysis of the possible influence of the distribution on the main parameters of the $\mathrm{KH}$ relaxation peak is made for the first time. It is shown that the distribution parameter $\beta$ determines the width, height, and asymmetry of the peak and also allows us estimate the relaxation strength from the peak height. In the paper, the algorithm of determining $\beta$ from the experimental data is given.

(3) When decreasing the mean grain size $\bar{d}$, the peak temperature $T_{P}^{\mathrm{KH}}$ and the peak width $\Delta\left(1 / \mathrm{T}_{0.5}^{\mp}\right)^{\mathrm{KH}}$ in the highly fragmented samples systematically decreased. The first effect is due to a decrease of the relaxation time $\tau$ at each temperature given, in other words, due to a decrease in the activation energy $U_{0}$ and/or the attempt period $\tau_{0}$. As the peak width should be inversely proportional to the activation energy $U_{0}$, the reason for the decrease of $\Delta\left(1 / \mathrm{T}_{0.5}^{\mp}\right)^{\mathrm{KH}}$ should be a decrease in $\tau_{0}$ when reducing the mean grain size.
(4) As a result of annealing, the peak temperature $T_{P}^{\mathrm{KH}}$ increases, i.e., a partial recovery of this parameter is observed. This effect is more pronounced in the most fragmented samples. This indicates that the structures of the submicrocrystalline samples prepared by the application of more complicated SPD schemes are less resistant to the heat treatment used.

(5) The relaxation strength values $\Delta_{\mathrm{M}}^{\mathrm{KH}}$ decrease after annealing in all the samples, i.e., this parameter of the $\mathrm{KH}$ relaxation, like the peak temperature, has a tendency to recovery. The decrease in the relaxation strength, apparently, is a consequence of the decrease in the dislocation density and, correspondingly, in the number of the operating relaxators.

(6) Unlike the peak temperature, the peak width does not show a recovery during annealing. Moreover, the value of $\beta$ continues to decrease. It means that, at least at the initial stage of recrystallization, the dispersion of relaxation times does not increase but even somewhat decreases, especially in the most fragmented samples. The decrease in $\beta$ with the mean grain size lowering may be explained by a narrowing of the dislocation length distribution at the expense of the longest dislocation segments $L>\bar{d}$. The next decrease in $\beta$ during annealing indicates further homogenization of the dislocation structure because of reducing the total dislocation density and lowering the level of internal stresses.

\section{Conflicts of Interest}

The authors declare that there are no conflicts of interest.

\section{Acknowledgments}

The authors are grateful to Drs. M. A. Tikhonovsky and P. A. Khaimovich for providing samples of zirconium and to Professor V. M. Chernov and Dr. S. N. Smirnov for their interest in the work and valuable discussions.

\section{References}

[1] Y. Estrin and A. Vinogradov, "Extreme grain refinement by severe plastic deformation: a wealth of challenging science," Acta Materialia, vol. 61, no. 3, pp. 782-817, 2013.

[2] T. G. Langdon, "Twenty-five years of ultrafine-grained materials: achieving exceptional properties through grain refinement," Acta Materialia, vol. 61, no. 19, pp. 7035-7059, 2013.

[3] E. D. Tabachnikova, A. V. Podolskiy, B. Bonarski et al., "Mechanical properties and microstructure evolution during deformation of ultrafine grained zirconium at low temperatures," Reviews on Advanced Materials Science, vol. 25, pp. 168-175, 2010.

[4] A. N. Velikodny, P. A. Khaimovich, M. A. Tikhonovsky, N. F. Andrievskaya, M. P. Starolat, and T. M. Tikhonovskaya, "Low-temperature semihydroextrusion of strongly deformed zirconium," High Pressure Physics and Technology, vol. 19, pp. 78-82, 2009, (in Russian).

[5] I. V. Kolodiy, N. F. Andrievskaya, V. N. Voyevodin, A. N. Velikodnyi, M. A. Tikhonovsky, and G. Y. Storozhilov, "Features of zirconium structure formation after severe plastic 
deformation," Problems of Atomic Science and Technology, vol. 5, pp. 98-104, 2015, (in Russian).

[6] E. N. Vatazhuk, P. P. Pal-Val, V. D. Natsik et al., "Lowtemperature acoustic properties of nanostructured zirconium obtained by intensive plastic deformation," Low Temp. Phys, vol. 37, no. 2, pp. 169-176, 2011.

[7] P. P. Pal-Val, M. Hamersky, Z. Trojanova, and P. Lukač, "Internal friction in a-zirconium polycrystals," Physica Status Solidi (a), vol. 125, no. 1, pp. K13-K16, 1991.

[8] V. D. Natsik, P. P. Pal-Val, and S. N. Smirnov, "Theory of a compound piezoelectric vibrator," Acoustical Physics, vol. 44, pp. 553-560, 1998.

[9] Z. Trojanova, P. Lukač, and P. P. Pal-Val, "Elastic and anelastic behaviour of zirconium polycrystals," Materials Science Forum, vol. 210-213, pp. 495-502, 1996.

[10] M. Koiwa and R. R. Hasiguti, "A theory of internal friction peak due to thermal unpinning of dislocations and its application to P1 peak in copper," Acta Metallurgica, vol. 13, no. 12 , pp. 1219-1230, 1965.

[11] L. J. Teutonico, A. V. Granato, and K. Lücke, “Theory of the thermal breakaway of a pinned dislocation line with application to damping phenomena," Journal of Applied Physics, vol. 35, no. 1, pp. 220-233, 1964.

[12] M. M. Savin and V. M. Chernov, "Interaction of dislocations with point defects in crystals with hexagonal close-packed structure," Physics of the Solid State, vol. 17, pp. 409-412, 1975, (in Russian).

[13] H. Wang, W. Li, T. Hao et al., "Mechanical property and damping capacity of ultrafine-grained $\mathrm{Fe}-13 \mathrm{Cr}-2 \mathrm{Al}-1 \mathrm{Si}$ alloy produced by equal channel angular pressing," Materials Science and Engineering: A, vol. 695, pp. 193-198, 2017.

[14] R. de Batist, Internal Friction of Structural Defects in Crystalline Solids, North Holland, Amsterdam, Netherlands, 1972.

[15] A. S. Nowick and B. S. Berry, "Lognormal distribution function for describing anelastic and other relaxation processes, I. Theory and numerical computations," IBM Journal of Research and Development, vol. 5, pp. 297-311, 1961.

[16] A. S. Nowick and B. S. Berry, Anelastic Relaxation in Crystalline Solids, Academic Press, New York, NY, USA, 1972.

[17] P. P. Pal-Val, M. Hamersky, Z. Trojanova, and P. Lukač, "Young's modulus of a-zirconium polycrystals as a function of temperature between 6 and 320 K," Physica Status Solidi (a), vol. 125, no. 1, pp. K17-K20, 1991.

[18] V. D. Natsik, E. N. Vatazhuk, P. P. Pal-Val, L. N. Pal-Val, and V. A. Moskalenko, "Observation of glass-like lowtemperature anomalies in the acoustic properties of nanostructured metals," Low Temperature Physics, vol. 39, no. 12, pp. 1078-1089, 2013.

[19] V. D. Natsik and Y. A. Semerenko, "Dislocation mechanisms of low-temperature internal friction in nanostructured materials," Low Temperature Physics, vol. 42, no. 2, pp. 138-150, 2016.

[20] A. S. Nowick and B. S. Berry, "Lognormal distribution function for describing anelastic and other relaxation processes. Data analysis and applications," IBM Journal of Research and Development, vol. 5, pp. 312-320, 1961. 


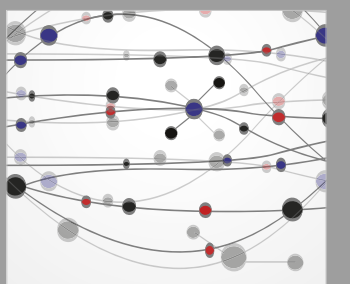

The Scientific World Journal
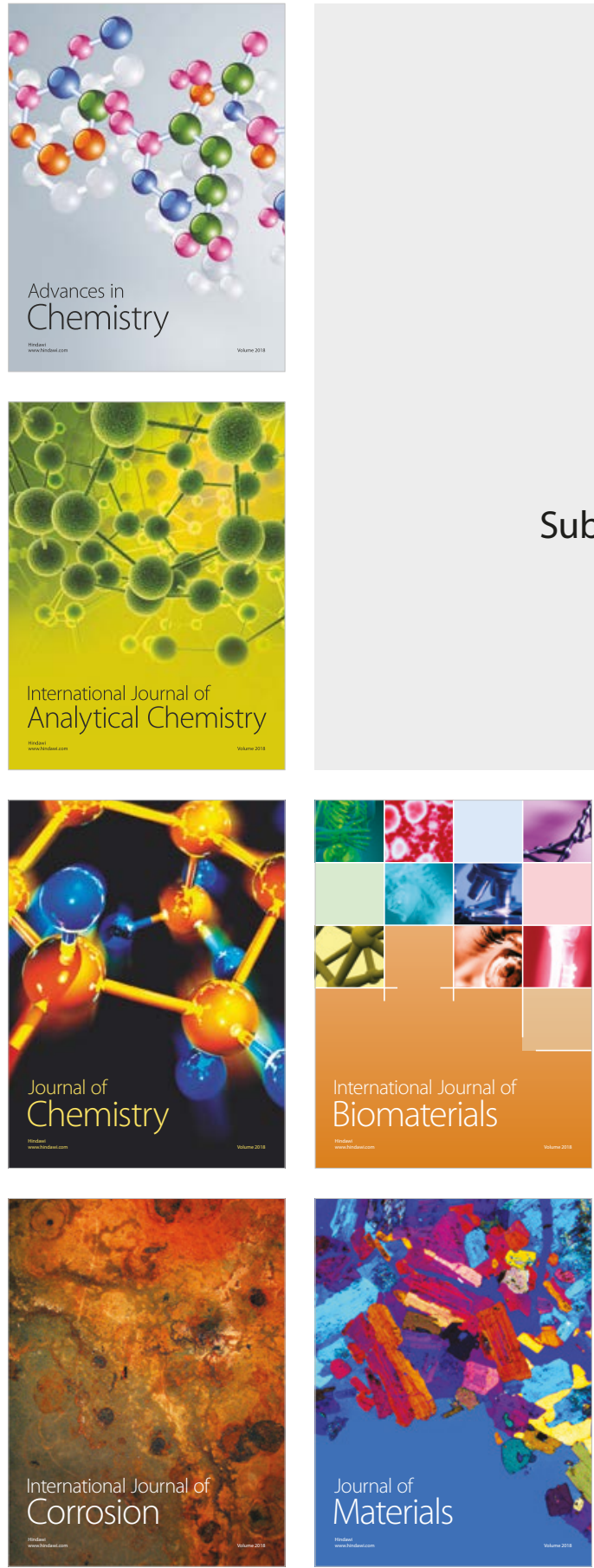

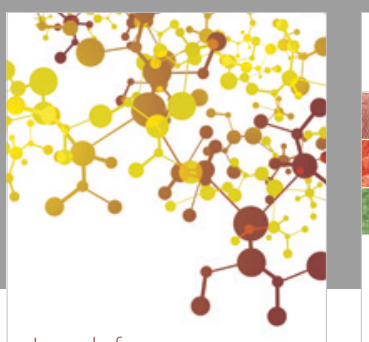

Journal of

Applied Chemistry
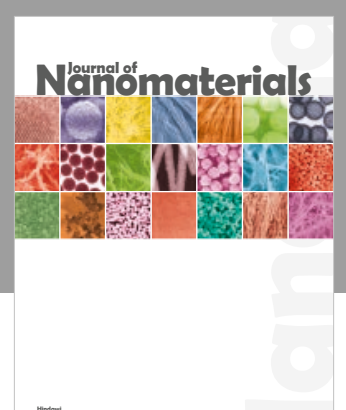

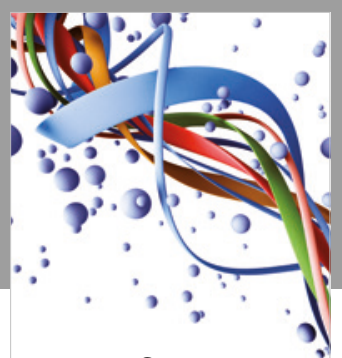

Scientifica

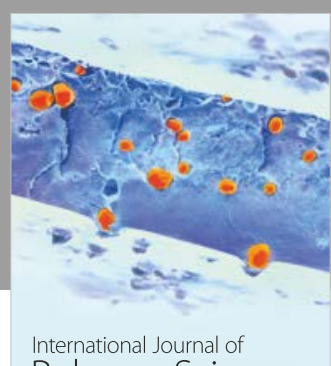

Polymer Science

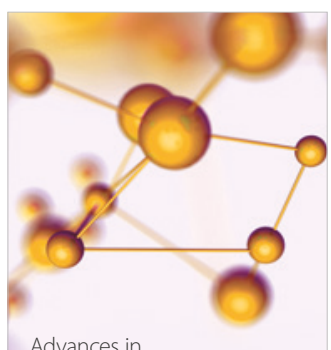

Physical Chemistry
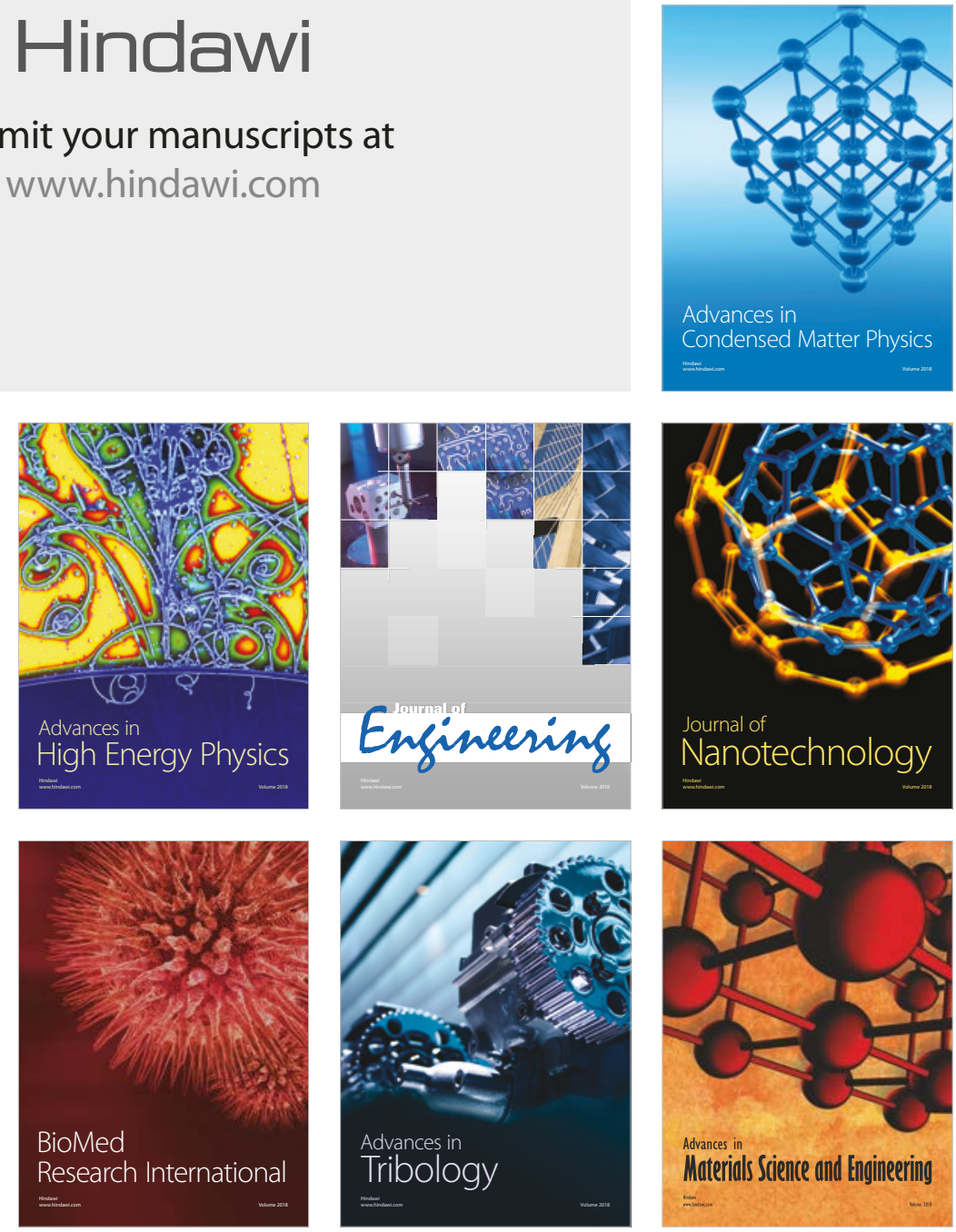